\title{
A Study on the Extracurricular Classes Education Model of Chinese Medicine and the Cultivation of Comprehensive Practical Ability of Chinese Students from Hong Kong, Macao, Taiwan and Overseas
}

\author{
Yahe Huang, Wenzhi Hao, Yu Wang, Xin Liu, Hoiyan Cheung \\ School of Chinese Medicine of Jinan University, Guangzhou, China \\ Email: HYH219@jnu.edu.cn
}

How to cite this paper: Huang, Y. H., Hao, W. Z., Wang, Y., Liu, X., \& Cheung, H. Y. (2018). A Study on the Extracurricular Classes Education Model of Chinese Medicine and the Cultivation of Comprehensive Practical Ability of Chinese Students from Hong Kong, Macao, Taiwan and Overseas. Creative Education, 9, 1389-1395.

https://doi.org/10.4236/ce.2018.99103

Received: June 27, 2018

Accepted: July 21, 2018

Published: July 24, 2018

Copyright $(9) 2018$ by authors and Scientific Research Publishing Inc. This work is licensed under the Creative Commons Attribution International License (CC BY 4.0).

http://creativecommons.org/licenses/by/4.0/

\begin{abstract}
Objective: To explore the extracurricular classes teaching mode and clinical practice ability of Chinese medicine in Hong Kong, Macao and Taiwan. Methods: A retrospective control study method was used to select the grade 2014 undergraduates and grade 2015 undergraduates of Jinan University as the research object, and the average performance of the two different modes in the traditional teaching and the new competition unit as a second class was added. Points, class pass rate, excellent rate and award-winning situation are compared to evaluate the effect of the new teaching model. Results: There is no significant difference between the 2014 level and the 2015 grades of Hong Kong, Macao and Taiwan. For the performance point, the 2015 Hong Kong, Macao and Taiwan students are increasing. There was no significant difference in the mean GPA between grade 2 and grade 2014 before the competition teaching model $(\mathrm{P}>0.05)$. After the competition teaching mode, the average GPA of the third grade of the former was much higher than that of the latter $(\mathrm{P}<0.05)$, compared with that of the overseas Chinese students of Hong Kong, Macao and Taiwan who received only the traditional Chinese medicine subject education in grade $2014(\mathrm{P}<0.05)$. From the point of passing rate and excellent rate, the pass rate and excellent rate of grade 1 were much higher than that of grade 2014, and the difference between them was statistically significant $(\mathrm{P}<0.05)$. As far as the award is concerned, the status of award-winning and project establishment in 2015 was higher than that in grade 2014, and the difference was statistically significant $(\mathrm{P}<0.05)$. Conclusion: Competition teaching is beneficial to the training of Chinese medicine learning and clinical
\end{abstract}


practice ability of Chinese students from Hong Kong, Macao, Taiwan and overseas.

\section{Keywords}

Extracurricular Classes Education, Clinical Practice, TCM

\section{Introduction}

In recent years, the number of students from Hong Kong, Macao, Taiwan and overseas Chinese has been increasing year by year in mainland colleges and universities; especially the number of Chinese medicine students in natural science has always occupied the first place (Fu, 2013). Its study aims at obtaining academic qualifications, obtaining certificates and promoting social status. Jinan University currently has the largest intake of foreign students in China, accounting for about half of the total number of students from 57 countries in the world and Hong Kong, Macao and Taiwan (Zhang, 2008). More than $80 \%$ of Chinese medical undergraduates come from Hong Kong, Macao, Taiwan and other parts of the world. Specific employment places result in employment where they need to be adapted to their places of origin. The traditional Chinese medicine practice is different from the mainland (She et al., 2010), and the traditional educational model cannot fully meet the requirements of the examination. Therefore, it is an urgent need to explore the training mode suitable for the students of Hong Kong, Macao and Taiwan for the cultivation of Chinese medical talents suitable for the local medical environment. Most overseas Chinese students like to participate in practical activities, show themselves in their activities, play their abilities and strengths, and enjoy the sense of achievement and belonging through this way. Therefore, the second classroom teaching, which includes basic theoretical study and clinical practice, is a new form of TCM teaching mode to explore its effectiveness in teaching practice.

\section{Methods}

\subsection{Sample}

By using the retrospective comparative study method, we selected grade 2014 TCM undergraduates of Jinan University and grade 2015 TCM undergraduates of Jinan University as research subjects. The indicators included: 1) Students from Hong Kong, Macau, Taiwan and overseas. 2) Prior to the undergraduate course, they had not received systematic Chinese medicine and clinical education. The indicators excluded: 1) students from mainland China. 2) The source of students is Hong Kong, Macao, Taiwan and overseas regions, but there have been systematic Chinese medicine and clinical education before.

\subsection{Study Method}

The grade 2015 TCM students of Hong Kong, Macau, Taiwanese and overseas 
receive only traditional Chinese medicine courses including Fundamental Theories of Traditional Chinese Medicine, TCM Diagnostics, Traditional Chinese Medicine, Pharmacology of Traditional Chinese Medical Formulae, and TCM internal medicine, studying in accordance with the school curriculum. Chinese Medical students from Hong Kong, Macau, Taiwan, and Taiwan in the 2016 year are studying in accordance with the school curriculum. At the same time, they will increase the new teaching model of the discipline competition, specifically developing clinical skills competitions, appreciation of traditional Chinese medicine works, Huangdi Neijing competition and Tongue image knowledge competition. The main contents include interlocution of basic knowledge of Chinese medicine, acupuncture skills competition, massage skills competition, clinical consultation and doctor-patient communication skills competition.

\subsection{Assessment Index}

The assessment index includes the student's GPA, pass rate and good rate, overall awards, and satisfaction of clinical departments in training learning.

\subsection{Statistical Methods}

All the data in this study were analyzed using statistical software SPSS 19.0. Measured data were expressed as mean \pm standard deviation $(\mathrm{x} \pm \mathrm{s})$, and the count data were expressed in proportion (\%). The t-test was used for the difference between the groups. The variance within the group was analyzed by variance. The count data were analyzed using the $\mathrm{x}^{2}$ test. $\mathrm{P}<0.05$ was considered statistically significant.

\section{Conclusion}

\subsection{Basic Data}

For grade 2014, there were 89 students from Hong Kong, Macao and Taiwan who met the inclusion criteria, of whom 75 were from Hong Kong, accounting for $84.3 \%$. 4 students from Macao, accounting for $4.5 \%$ of the total; Taiwan has 9 students, accounting for $10.1 \%$ of the total. 1 overseas Chinese (Singapore), accounting for $1 \%$; for grade 2015, there were 39 overseas students from Hong Kong, Macao and Taiwan, who met the inclusion criteria. Among them, there were 33 students from Hong Kong, accounting for $84.6 \%$. Macau has 3 students, accounting for $7.7 \%$. Two Taiwanese students account for $5 \%$. One overseas Chinese (Columbia), accounting for $3 \%$. There was no statistically significant difference in the overall composition of students from Hong Kong, Macao and Taiwan $(\mathrm{P}>0.05)$ shown in Table 1.

\subsection{Average GPA}

At the end of the study, the average GPA of Grade 2014 students from Hong Kong, Macao, Taiwan and overseas Chinese was compared with that of Grade 2015 students from Hong Kong, Macao and Taiwan. The statistical results are 
Table 1. Comparison of students' Composition ratio between grade 2014 and grade 2015 $[\mathrm{n}(\%)]$.

\begin{tabular}{ccccccc}
\hline \multirow{2}{*}{ Group } & \multirow{2}{*}{ Total } & \multicolumn{4}{c}{ Students' Composition Ratio } & P Value \\
\cline { 3 - 7 } & & Hong Kong & Macau & Tai Wan & Overseas & \\
\hline GRADE 2014 & 89 & $75(84.3 \%)$ & $4(4.5 \%)$ & $9(10.1 \%)$ & $1(1 \%)$ & $>0.05$ \\
GRADE 2015 & 39 & $33(84.6 \%)$ & $3(7.7 \%)$ & $2(5 \%)$ & $1(3 \%)$ & \\
\hline
\end{tabular}

shown in Table 2. Compared with the overseas Chinese students in Hong Kong, Macao and Taiwan who received only traditional Chinese medicine education in 2014, the average GPA of the former was much higher than that of the latter, and the difference between them was statistically significant $(\mathrm{P}<0.05)$.

\subsection{High Quality Academic Science and Technology Activities Are the Important Pillar of the Second Class of TCM Students}

At the end of the study, a comparison was made between the overall pass rate and the excellence rate of the 2014 grade Hong Kong, Macao, Taiwan and overseas Chinese students and the 2015 grade Hong Kong, Macao and Taiwan overseas Chinese students. The statistical results are shown in Table 3. The data in the table show that the 2015 Hong Kong, Macao, and Taiwan overseas Chinese students have increased the competition teaching mode. There was no significant difference in pass rate and excellent rate between grade 2014 and grade 2014 ( $\mathrm{P}>0$ 0. 05). After the competition teaching mode, the passing rate and excellent rate of the former were much higher than those of the overseas Chinese students from Taiwan, Hong Kong, Macao and Taiwan who received only the traditional Chinese medicine subject education in grade 2014. The difference between them was statistically significant $(P<0.05)$.

\subsection{Comparison of Awards}

At the end of the study, a comparison was made between grade 2014 Hong Kong, Macao, Taiwan and overseas Chinese students and grade 2015 Hong Kong, Macao, Taiwan and overseas Chinese students (including the project proposal). The statistical results are shown in Table 4. The data in the table show that the number of Hong Kong, Macao, Taiwan and overseas Chinese students (including the project proposal) has increased significantly compared with the 2014 grade, and the difference between them is statistically significant $(\mathrm{P}<$ $0.05)$.

\section{Discussion}

\subsection{Stimulate Learning Enthusiasm and Improve Clinical Speculative Ability}

Traditional Chinese medicine (TCM) is a combination of theory and practice. Clinical skill contest is not only the carrier of talent training, but also an effective means to detect the quality of talent training. The expansion of enrollment and 
Table 2. Comparison of students' GPA between grade 2014 and grade 2015.

\begin{tabular}{cccc}
\hline \multirow{2}{*}{ Group } & Total & \multicolumn{2}{c}{ Average GPA } \\
\cline { 3 - 4 } & & Second Grade & Junior Class \\
\hline GRADE 2014 & 89 & 2.50 & 2.26 \\
GRADE 2015 & 39 & 2.55 & 2.50 \\
P Value & - & $>0.05$ & $<0.05$ \\
\hline
\end{tabular}

Table 3. Comparison of overall pass rate and excellent rate between grade 2014 and grade 2015.

\begin{tabular}{|c|c|c|c|c|c|}
\hline \multirow{2}{*}{ Group } & \multirow{2}{*}{ Total } & \multicolumn{2}{|c|}{ Overall Pass Rate } & \multicolumn{2}{|c|}{ Overall Excellence Rate } \\
\hline & & Second Grade & Junior Class & Second Grade & Junior Class \\
\hline GRADE 2014 & 89 & $37 \%$ & $49 \%$ & $12.35 \%$ & $7.8 \%$ \\
\hline GRADE 2015 & 39 & $53 \%$ & $35 \%$ & $28.2 \%$ & $28.2 \%$ \\
\hline P Value & - & $>0.05$ & $<0.05$ & $>0.05$ & $<0.05$ \\
\hline
\end{tabular}

Table 4. Comparison of students' Award between grade 2014 and grade 2015 [n(\%)].

\begin{tabular}{ccccccc}
\hline & & \multicolumn{3}{c}{ Awards(including project proposal) } & P Value \\
\cline { 3 - 6 } Group & Total & School level & Provincial level & $\begin{array}{c}\text { Challenge } \\
\text { cup }\end{array}$ & $\begin{array}{c}\text { University student } \\
\text { entrepreneurship } \\
\text { plan }\end{array}$ \\
\hline $\begin{array}{c}\text { GRADE } \\
\text { 2014 }\end{array}$ & 89 & 0 & 0 & 0 & 0 & $<0.05$ \\
$\begin{array}{c}\text { GRADE } \\
2015\end{array}$ & 39 & 3 & 3 & 4 & 1 & \\
\hline
\end{tabular}

the present situation of medical environment result in less chance of operation, limited clinical thinking unskillful operation of basic diagnosis and treatment (Zhang, 2006). The intensive training of clinical skills in the contest of TCM clinical skills enhances the proficiency of skill operation, and can stimulate students' enthusiasm for learning and help to improve clinical skills rapidly. This study shows that the competition into the extracurricular class, through clinical skills practice, doctor-patient relationship communication competition, can stimulate the enthusiasm of students to learn, improve their clinical practice.

\subsection{Strengthen the Learning of Classical Chinese Medicine and Improve the Level of TCM Theory}

Because the students from Hong Kong, Macao, Taiwan and overseas Chinese have the same learning background before entering school, the Chinese culture of most students is weak; the comprehension of traditional Chinese medicine terminology is often more difficult; and it is difficult for them to learn the classical course of traditional Chinese medicine (Chen et al., 2009). However, the key position of traditional Chinese medicine classics in Chinese medicine education is unspoken, because the development and innovation of any subject should 
be based on deep theoretical foundation. Therefore, the development of traditional Chinese Medicine Classic Competition to promote teaching will play a great role in promoting the level of TCM theory (Feng \& Huang, 2018).

\subsection{High Quality Academic Science and Technology Activities Are the Important Pillar of the Second Class of TCM Students}

In the whole campus culture, the cultural achievements provided by academic activities: world view, methodology, scientific theory, code of conduct, technology and academic spirit, academic atmosphere, etc., gradually permeate all aspects of campus cultural activities, and play a positive role in guiding, supporting, standardizing, promoting and so on. At the same time, the innovation of academic science and technology and academic research bring up talents with innovative consciousness, and they are the main bodies of campus cultural activities, and directly participate in the construction of campus culture, which is the motive force of campus culture construction (Guo et al., 2017). Therefore, academic activities play a leading role in the construction of the second class of TCM students and determine the level of the second classroom construction. In this study, the results show that after the introduction of competition mechanism into the second classroom, the achievement points, pass rates, excellence rates and awards of overseas Chinese students in Hong Kong, Macao, and Taiwan have improved significantly, reflecting the important position of high quality academic and scientific activities.

To sum up, holding all kinds of competitions provides a platform for exchange and display for Hong Kong, Macao, Taiwan and overseas Chinese, and experiences the fun of competition, which is conducive to promoting the psychological motivation of learning and building a good style of study. At the same time, the fierce competitive competition can also improve the students' overall quality, exercise the ability of coordination and cooperation among the members of the participating teams, enhance the emotional exchange between students, and help students to grow up in an all-round way (Xu et al., 2009). The model of introducing competition mechanism into the second classroom education of TCM and the cultivation of clinical practice ability is worth popularizing.

\section{Outlook}

In this study, only the 2014 and 2015 Hong Kong, Macao and Taiwanese overseas students' GPA, pass rates and awards were compared, but not the multi-center and multi-grade students. In the next step, we will cooperate with different universities and departments of traditional Chinese medicine to promote the application of competition model in the second classroom of Chinese medicine and evaluate it more accurately and objectively.

\section{References}

Chen, L. X., Zhang, H. F., \& Zhu, L. Y. (2009). A Study of Differences in Learning Styles between Hong Kong, Macao, Taiwan and Overseas Chinese Students. Higher Educa- 
tion Exploration, 6, 104-107.

Feng, Q., \& Huang, L. (2018). Through Clinical Skills Contest Training Outstanding Medical Talents Strategy Analysis. Education Teaching Forum, 1, 217-218.

Fu, P. (2013). Research on the Training Mode of Chinese Medicine Personnel in Hong Kong, Macao and Taiwan. Contemporary Medicine Forum, 1, 89-90.

Guo, K. H., He. J. F., \& Tao, X. Z. (2017). Quality Assurance and Reflection on the Practice Teaching of Medical Talents Training in Zhongshan University. Higher Medical Education in China, 12, 67-68.

She, S. F., Lu, D. Y., \& Liu, F. B. (2010). People-Oriented, Because of the Ability to Teach Hong Kong, Macao and Taiwan Students' Clinical Teaching Experience. Chinese Medicine Modern Distance Education of China, 8, 31-32.

Xu, J., Gao, J., \& Mai, J. Y. (2009). The Relationship between Self-Harmony and Mental Health of Hong Kong, Macao and Taiwan Students from TCM Universities. In Proceedings of the Sixth Annual Conference of the International Chinese Academy of Applied Psychology (pp. 308-318). Dandong.

Zhang, S. J. (2008). Study on the Study Mode of Hong Kong and Macao Students in the Mainland. Guangzhou: Jinan University.

Zhang, X. Y. (2006). Practice and Consideration on Academic and Cultural Contraction for High Quality College Students on Campus. ECM, 25, 22-25. 